\title{
Simulation numérique des réacteurs gaz-liquide utilisés en traitement de l'eau
}

\author{
Numerical simulation of gas-liquid reactors used in water treatment
}

par Z. Do-Quang, A. Cockx

CIRSEE, Lyonnaise des Eaux, Le Pecq, France

\author{
A. Line, M. Roustan
}

LIPE, EA833, INSA-GPI, Toulouse, France

The main objective in water industry is to ensure the adequate treatment process, guarantee its maximum efficiency and contain investment and operating costs. Due to the increasing power of computers, Computational Fluid Dynamics modelling approach becomes an efficient tool to reach this objective.

This paper resumes some applications of the CFD modelling to the study of the hydrodynamic and mass transfer performance of single-and multi-phase biological and/or chemical reactors used in water and wastewater treatment. Numerical simulations together with experimental measurements have been performed on laboratory and full-scale plants in order to gain better understanding of the physical phenomena (mixing, interfacial transfer) occurring in these systems in order to improve their design and process efficiency. The exemples described in the paper allow to highlight the feasibility and the provisional limitations of the CFD modelling tools. In most of the cases complementary work is necessary to be carried on in order to improve the mathematical models related basically to a better knowledge of the physical parameters controlling the hydrodynamics of the reactors.

\section{I $\square$ INTRODUCTION}

La préoccupation croissante en matière de santé publique ainsi que les progrès récents réalisés dans le domaine de la microbiologie et les techniques analytiques ont eu pour conséquence de renforcer la réglementation sur les procédés de traitement des eaux pour la potabilisation ou l'épuration des eaux usées. Cela nous amène à considérer en même temps des objectifs de qualité multiples et souvent contradictoires afin de répondre aux exigences tant sur le plan du risque sanitaire (en garantissant $100 \%$ fiabilité) que de celui du confort client (goûts et odeurs). Pour arriver à maîtriser l'efficacité de l'ensemble des chaînes de traitement il est primordial de comprendre et de décrire correctement les phénomènes conduisant à l'évolution de la qualité de l'eau au cours de chaque étape. Compte tenu de la complexité des interactions entre les différents paramètres de qualité et les différents procédés mis en œuvre, l'unique voie de l'expéri- mentation et de l'utilisation de corrélations empiriques ne suffise plus à définir le mode de fonctionnement optimal et la gestion des périodes de crise. Seule la modélisation mathématique à travers des outils adaptés permet d'accéder à la maîtrise totale de l'évolution de la qualité de l'eau à travers une chaîne de traitement [17].

\section{II $\square$ PRÉSENTATION DU CODE ASTRID}

Le code de calcul ASTRID analyse et simule des écoulements tridimensionnels diphasiques turbulents à inclusions dispersées. La présentation détaillée du code a été publiée par Simonin et Viollet $[15,16]$. Leur modèle est basé sur la conservation de la masse et de la quantité de mouvement exprimée pour chaque phase : c'est le modèle à deux fluides. Le transfert interfacial de quantité de mouvement est modé- 
lisé pour des inclusions sphériques et pour une population de taille homogène et s'exprime par les forces de traînée, de lift et de masse ajoutée. La modélisation de la turbulence dans la phase continue est issue d'un modèle classique à deux équations $(k, \varepsilon)$ et dans la phase dispersée le modèle de Tchen a été adapté. La résolution donne en tout point géométrique et à chaque instant les champs de vitesse et de turbulence dans chaque phase, ainsi que le taux de présence local de ces phases.

\section{EXEMPLES DE RÉSULTATS DE SIMULATION NUMÉRIQUE}

\subsection{Approche théorique pour la modélisation du trans- fert interfacial de quantité de mouvement}

Différents contacteurs polyphasiques utilisés en production d'eau potable ou en traitement d'eau résiduaire urbaine sont présentés. Les paramètres qui contrôlent les performances de ces systèmes sont l'hydrodynamique des différentes phases, le transfert de masse gaz-liquide (impliquant la distribution des phases et l'aire interfaciale) et la cinétique des réactions chimiques et biologiques. L'application du concept de la Mécanique des Fluides Numériques est un nouveau challenge. Dans ce domaine beaucoup de questions relatives à la modélisation du transport interfacial et au transfert de masse local se posent. On rencontre encore beaucoup de difficultés également lors de l'estimation du diamètre de bulles et de l'aire interfaciale ainsi que concernant la modélisation de la turbulence de la phase continue. L'amélioration de la qualité des prédictions numériques dépend de la meilleure compréhension des phénomènes physiques locaux de base cités plus haut. Par conséquent l'expérience reste incontournable dans ce domaine pour améliorer les modèles et valider les simulations. La modélisation des écoulements diphasiques est assez complexe [2]. Dans cet article nous nous limitons à l'analyse de deux paramètres principaux : le diamètre de bulles équivalent et le coefficient de traînée. Ce choix est conditionné par le type d'écoulement à bulles auquel nous nous intéressons, c'est-à-dire l'écoulement dans lequel la phase dispersée contrôle 1'hydrodynamique [4]. Dans les simulations pour différentes valeurs du coefficient de traînée et différentes formes et tailles des bulles on obtient différentes distributions des fractions volumiques des phases.

La rétention gazeuse est le paramètre qui doit être prédit avec beaucoup de précision car il contrôle l'aire interfaciale volumique. L'aire interfaciale volumique projetée est donnée par:

$$
a=\frac{1,5 \alpha}{d_{b} E^{2,3}}
$$

où $\alpha$ est la fraction volumique locale du gaz, $d_{b}$ est le diamètre de la bulle sphérique ayant le même volume et $E$ est l'excentricité dans le cas d'une bulle ellipsoïdale. $\alpha$ est prédite par un modèle et comparée aux mesures expérimentales.

En ce qui concerne le coefficient de traînée, différentes expressions théoriques ont été proposées dans le cas d'une bulle isolée immergée dans un champ uniforme pour le fluide à l'infini ou par simulation directe $[9,10]$. Dans la mesure où il n'existe pas de modèle capable de prédire le diamètre des bulles dans n'importe quel type de réacteur, pour les simulations nous avons considéré le diamètre des bulles équivalent constant et donné par l'expérience. Dans le cas de présence des bulles ellipsoïdales la valeur expérimentale de l'excentricité a été introduite dans le code. L'expression du coefficient de traînée $C_{D}$ selon Moore $[12,13]$ est :

$$
C_{D}=\frac{48}{R e}\left(1-\frac{2,21}{R e^{1,2}}\right)
$$

où Re est le nombre de Reynolds de la bulle. Il a été confirmé que ce coefficient pour une bulle est plus faible que pour une inclusion rigide $\left(C_{D}=0,44\right)$. En cas de distorsion des bulles, Moore propose un ensemble de relations analytiques afin d'exprimer le coefficient de traînée en fonction du nombre de Reynolds de la bulle, du nombre de Morton et de l'excentricité.

\section{- 3.2 Réacteur biologique à lit circulant TURBO (air-lift)}

Les nouveaux standards européens pour l'élimination simultanée de la pollution l'azotée, carbonée et phosphorée ont amené les industriels à la réhabilitation des stations d'assainissement existantes et au design de nouvelles technologies intensives avancées $[7,11]$. Les réacteurs à cultures fixées (lits fixés ou lits fluidisés) permettant de développer des biofilms minces très actifs sont apparus comme une alternative au procédé conventionnel de cultures libres (bassins d'aération) à cause de leur compacité et leur cinétique de nitrification élevée. Essentiellement deux types de procédés se retrouvent parmi les réacteurs polyphasiques à cultures fixées : les lits fluidisés et turbulés, où la mise en suspension des matériaux solides est assurée par l'unique contribution d'un débit de gaz ou de liquide et les réacteurs à lit circulant de type air-lift triphasique à boucle interne ou à boucle externe. L'optimisation de ces procédés passe par la maîtrise de leur hydrodynamique en écoulement diphasique gazliquide.

Dans les lits fluidisés ou les lits circulants ayant l'avantage de ne pas présenter de problèmes de colmatage, la force motrice du procédé est l'injection. Le pilote de laboratoire airlift est un réacteur de type cuve rectangulaire $(3 \mathrm{~m}$ en hauteur, $0,5 \mathrm{~m}$ en largeur) divisé en deux parties par une paroi interne. De l'air est injecté au moyen de membranes assymétriquement par rapport à la paroi en bas du réacteur et le déséquilibre de rétention gazeuse qui apparaît entre les deux côtés est à l'origine de la mise en mouvement du liquide.

Les simulations ont été conduites sur un écoulement en deux dimensions. Deux positions du distributeur ont été étudiées : au fond du réacteur et surélevé de $20 \mathrm{~cm}$ par rapport

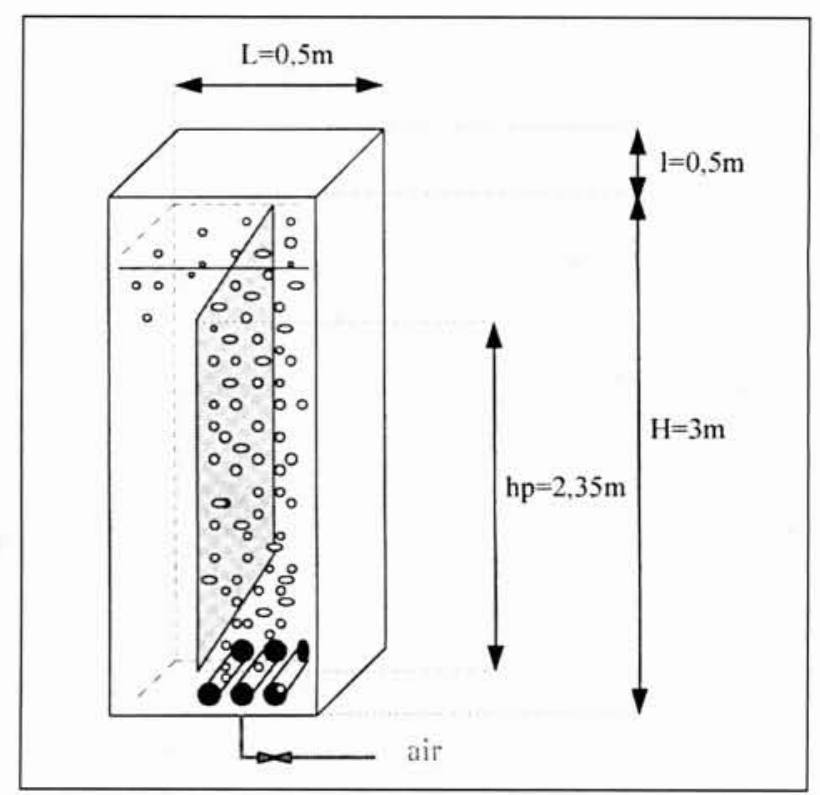

1. Schéma du pilote expérimental. 


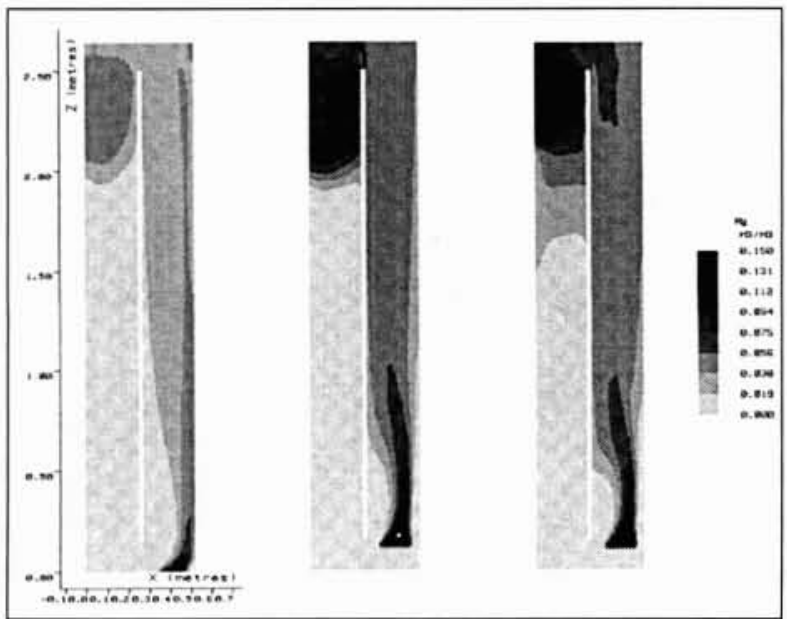

2. Distributions spatiales de la rétention gazeuse dans un plan 2D simulées avec le code ASTRID; test de différentes relations de fermeture sur le coefficient de traînée et la sensibilité des conditions aux limites.

au fond. Dans toutes les simulations le débit de gaz est de $9 \mathrm{~kg} / \mathrm{s}$ (vitesse débitante $U g=1,7 \mathrm{~cm} / \mathrm{s}$ ).

Les résultats de la simulation montrent la distribution spatiale du gaz dans le réacteur. La rétention moyenne du gaz dans un plan horizontal a été mesurée à différents niveaux à la fois dans la partie montante et dans la partie descendante du réacteur $[3,7]$. Les valeurs calculées numériquement ont été moyennées dans les mêmes points. La comparaison des résultats numériques et l'expérience sont représentés sur la figure 4. L'estimation de la distribution du gaz est satisfaisante. Les résultats montrent que puisque la population de bulles est monodiamètre, la fraction volumique est constante dans la partie ascendante de l'airlift sauf près du distributeur où l'accélération des vitesses due au rétrécissement diminue le taux de présence de gaz. Dans la partie descendante, une recirculation liquide emprisonne les bulles qui ont une très faible vitesse. Le taux de présence est donc très élevé dans cette zone supérieure et nul plus bas car les vitesses liquides sont alors trop faibles. Les profils longitudinaux numériques et expérimentaux sont très proches et la simulation a permis aussi bien de retrouver les valeurs moyenne de rétention que la zone d'aération descendante ce qui, a priori, nous paraissait plus difficile. Le profil horizontal (selon X) de vitesse liquide dans la zone non-aérée et dans la partie inférieure de l'écoulement descendant a été mesurée à partir de la P.I.V. (Particle Image Velocimetry), technique de mesure instantanée de champ de vitesse par plan laser [6]. Les résultats satisfaisants de la comparaison entre les vitesses liquide mesurées et simulées montrent qu'une bonne estimation de la distribution du gaz dans le réacteur mène à une bonne estimation de la circulation liquide induite $[3,4,5]$.

A partir des résultats hydrodynamiques il est possible de simuler la dispersion axiale dans le réacteur air-lift [4] et déterminer le nombre de Peclet correspondant défini comme :

$$
P e=\frac{U_{Z} \cdot L}{E_{Z L}}
$$

Côté expérimental, il est possible de déduire le nombre de Peclet à partir de la courbe de variation temporelle de la concentration du traceur introduit dans le système. Cette courbe expérimentale peut être approché par le modèle de Voncken :

$$
\frac{C}{C 0}=\sqrt{\frac{P e}{4 \Pi \theta}} \sum_{j=0}^{p} \exp \left[-\frac{P e}{4 \theta}(j-\theta)^{2}\right]
$$

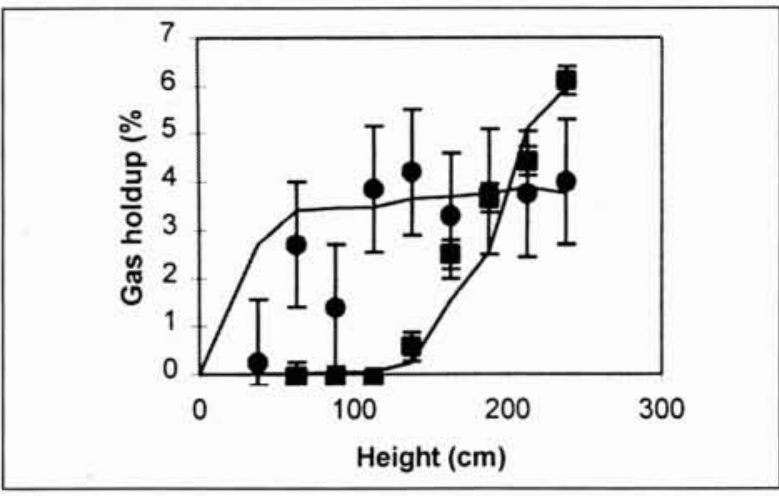

3. Comparaison du profil longitudinal expérimental de la rétention gazeuse dans le riser $(\bullet)$ et le downcomer (E) et le calcul numérique (_ $)$.

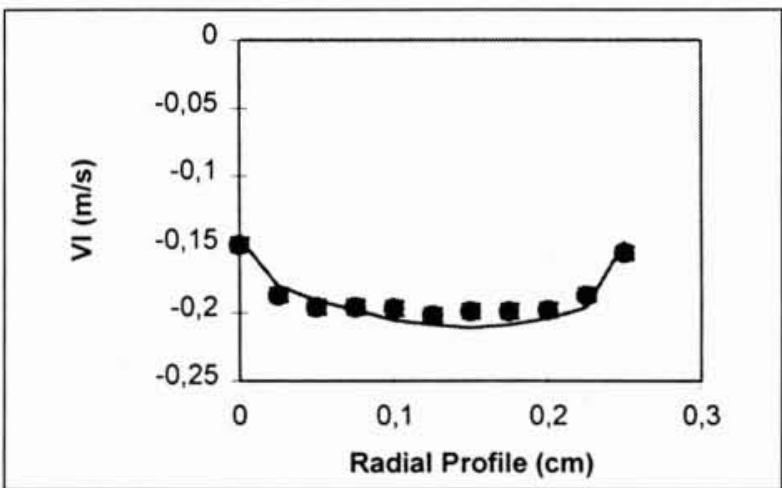

4. Comparaison des profils expérimental $(\bullet)$ et numérique $\left({ }_{(}\right)$de la vitesse liquide dans la zone nonaérée du downcomer.

où $C=$ concentration au temps $t, C 0=$ concentration dans le réacteur après homogénéisation et $\theta=t / t c$ temps normé par le temps de circulation.

Sur la figure 5 est représentée la courbe de Voncken qui prend correctement en compte les points expérimentaux. Le nombre de Peclet expérimental et le coefficient de dispersion sont déterminés à partir de l'expérience :

$\left\{\begin{array}{l}t c=25 s \\ P e=30\end{array}\right\} V c \approx 0,2 \mathrm{~m} / \mathrm{s}$

La figure 6 montre la confrontation entre le profil de concentration simulé par ASTRID et celui calculé à partir du

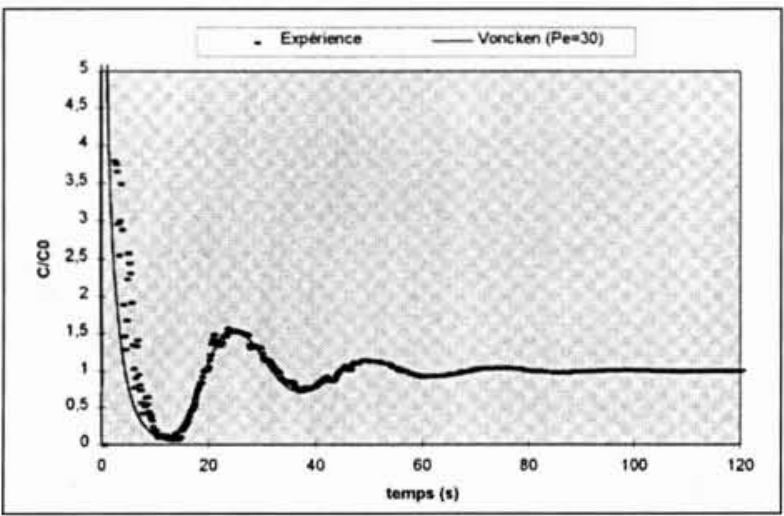

5. Profil de concentration mesuré comparé au modèle de Voncken $(Q g=9 \mathrm{~kg} / \mathrm{s})$. 


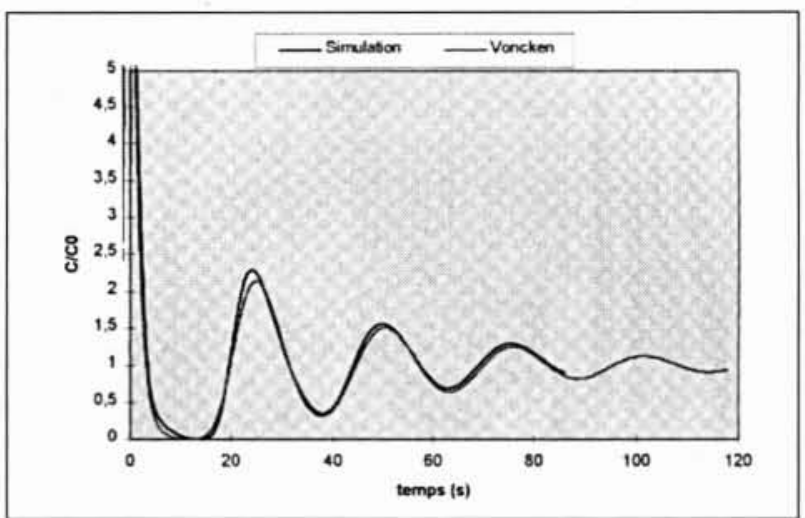

6. Profil de concentration simulé comparé au modèle de Voncken.

modèle de Voncken. Les mêmes variables que précédemment sont alors déterminées :

$\left\{\begin{array}{l}t c=25 s \\ P e=50\end{array}\right\} V c \approx 0,2 \mathrm{~m} / \mathrm{s}$

L'écart entre les deux valeurs de $E_{\mathrm{ZL}}$ provient du fait que dans les simulations numériques l'écoulement a été considéré comme bi-dimensionnel alors qu'en réalité il est tridimensionnel. De plus, le modèle de turbulence ne prend pas en compte l'influence de la phase dispersée sur la phase continue.

\subsection{Bassins d'aération}

Dans les bassins d'aération, utilisés en procédés biologiques aérobies d'épuration des eaux, le nombre, le type et l'empla- cement des systèmes d'aération restent un problème complexe [14]. Pour améliorer la compréhension des recirculations liquides induites par les diffuseurs de gaz, nous avons simulé un bassin d'aération très simplifié. Le chenal est en deux dimensions et nous avons placé un unique diffuseur d'air au fond soumis à une vitesse horizontale de l'eau $U$ l homogène sur la hauteur totale du chenal.

Cette vitesse de circulation est générée par les agitateurs placés dans le bassin et peut varier selon leur vitesse de rotation. Les résultats des simulations montrent que le panache de bulles monte verticalement si $U \mathrm{I}=0$. Dans ce cas les bulles créent des recirculations liquides qui ont tendance à diminuer le taux de présence de gaz. Ce panache s'incline ensuite d'autant plus que $U l$ augmente. A partir d'une vitesse liquide critique $(U l=0,25 \mathrm{~m} / \mathrm{s})$, les rouleaux de circulation sont cassés et le taux de présence de gaz n'est plus diminué, ce qui augmente l'aire interfaciale volumique et donc le transfert d'oxygène, de 30 à $40 \%$ plus efficaces dans des bassins munis d'agitateurs à flux horizontal. Il faudrait maintenant valider ces résultats quantitativement par des mesures de taux de gaz et de vitesses des liquides. A partir des simulations il est possible de calculer l'aire interfaciale moyenne. Les résultats sont résumés dans le tableau 1. La présence d'une vitesse horizontale liquide fait augmenter l'aire interfaciale jusqu'à $40 \%$ par rapport à la situation sans vitesse liquide.

Tableau 1. - Aire interfaciale simulée pour différentes vitesses de liquide.

\begin{tabular}{|c|c|c|}
\hline $\begin{array}{c}\text { Vitesse } \\
\text { liquide }(\mathrm{m} / \mathrm{s})\end{array}$ & $\begin{array}{c}\text { Aire interfaciale } \\
\mathrm{a}\left(\mathrm{m}^{2} / \mathrm{m}^{3}\right)\end{array}$ & $\begin{array}{c}\text { Rapport } \mathrm{a} / \mathrm{a} \\
(0 \mathrm{~m} / \mathrm{s})\end{array}$ \\
\hline 0 & 13,9 & 1 \\
0,1 & 16,8 & 1,2 \\
0,2 & 18,4 & 1,3 \\
0,3 & 19,6 & 1,4 \\
0,4 & 19,6 & 1,4 \\
\hline
\end{tabular}

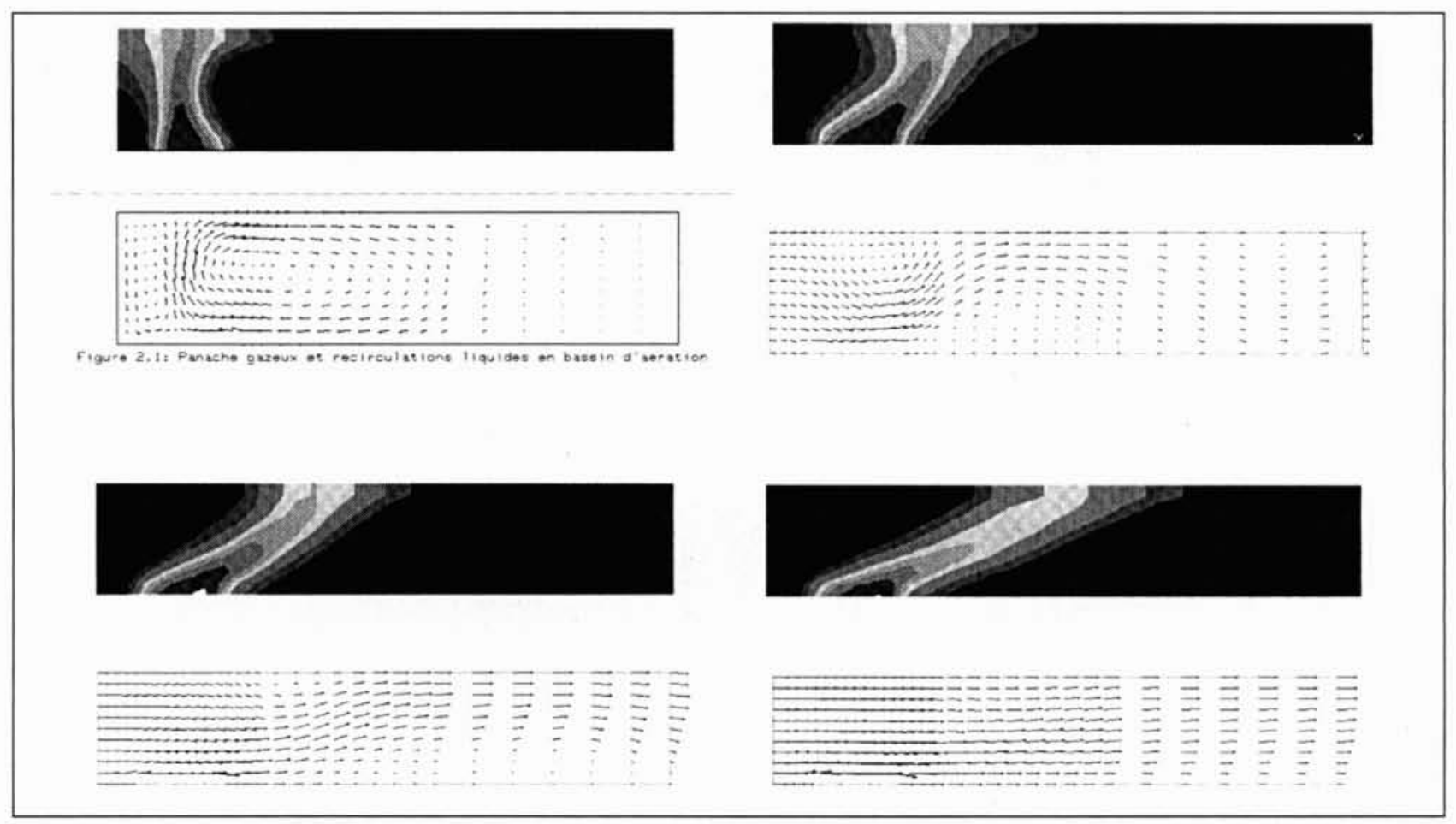

7. Distribution spatiale de la rétention gazeuse et champ vectoriel de la vitesse liquide dans différents cas de figure : $U l=0 ; 0,1 ; 0,2$ et $0,3 \mathrm{~m} / \mathrm{s}$, vitesse de gaz $=0,01 \mathrm{~m} / \mathrm{s}$ (couleur bleu rétention gazeuse $=0 \%$, couleur rouge rétention gazeuse $=7 \%$ ). 


\subsection{Réacteurs d'ozonation}

Sur les chaînes de traitement d'eau potable l'objectif principal de qualité est de garantir l'efficacité maximale du traitement en assurant une désinfection adéquate dans des conditions de formation minimisée de sous-produits et d'élimination des goûts et des odeurs. Voilà pourquoi la préoccupation majeure est le choix de la géométrie optimale et les conditions opératoires afin d'obtenir les meilleures performances hydrodynamiques et de transfert de masse qui déterminent l'efficacité de l'élimination des micro-organismes tels que les bactéries, les virus, les kystes protozoaires (Giardia et Cryptosporidium). Un exemple d'un réacteur d'ozonation multicompartiment est donné à la figure 8. Les caractéristiques géométriques sont : longueur $=13 \mathrm{~m}$, hauteur $=4,75 \mathrm{~m}$, largeur $=4,2 \mathrm{~m}$. Le débit d'eau peut varier entre 1000 et $2000 \mathrm{~m}^{3} / \mathrm{h}$. Le débit de gaz ozoné est constant $\left(160 \mathrm{Nm}^{3} / \mathrm{h}\right)$ mais la concentration d'ozone dans le gaz est ajustée en fonction de la variation du débit d'eau et de la demande en ozone de l'eau.

Les hypothèses de base du modèle numérique et les paramètres de simulation sont résumés ci-après :

- maillage tridimensionnel composé de 260000 nœuds

- taux de présence maximum pour la phase dispersée = $10 \%$

- pas de modèle de coalescence ou de rupture des bulles

- phase continue : modèle de turbulence $k-\varepsilon$

- phase dispersée : modèle de l'équilibre local

- pas de temps $=0,05 \mathrm{~s}$

- pas d'espace $=0,02 \mathrm{~m}$

- vitesse d'eau à l'entrée $=0,4 \mathrm{~m} / \mathrm{s}$

- vitesse du gaz à l'entrée $=0,1 \mathrm{~m} / \mathrm{s}$

- diamètre de bulles $=3 \mathrm{~mm}$
- coefficient de traînée pour les bulles $C_{D}=1$

- taux de présence du gaz à l'entrée $=0,15$.

Les distributions spatiales du taux de présence de gaz ont été calculées pour les différentes configurations géométriques (fig. 8 et 9). Le gaz n'est pas introduit de façon homogène dans l'ensemble du réacteur. Sa distribution est choisie en fonction des réactions chimiques spécifiques se produisant dans le système. Différentes dispositions des parois internes et des diffuseurs ont été étudiées afin de trouver la configuration optimale pour atteindre le niveau de désinfection le plus élevé. L'hydrodynamique du système est imposée essentiellement par l'écoulement de l'eau qui dépend du nombre et de la position de chicanes. L'augmentation du nombre de chicanes améliore à la fois le caractère piston global de l'écoulement et l'efficacité du transfert gazliquide. D'une part, les parois servent de guide pour l'eau dans le réacteur et ainsi limitent les probabilités de court-circuit ou formation de zones mortes. D'autre part elles empêchent la formation de zones de recirculation et de mélange induits par les bulles à l'intérieur des compartiments de diffusion de gaz.

L'introduction de chicanes rétrécit les compartiments et crée un écoulement co-courant ou contre-courant entre les deux phases. En l'absence de chicanes les compartiments deviennent plus larges et les circulations liquides induites par les bulles sont intensifiées. Le volume entier est occupé par ces zones parfaitement mélangées formées suite à l'interaction entre l'écoulement de l'eau et du gaz. Dans ces cas un niveau de désinfection plus faible est observé. Le coefficient de transfert global dépend de l'aire interfaciale de transfert qui dépend de la rétention globale du gaz et de la taille des bulles. Les simulations numériques ainsi que les mesures expérimentales de coefficient de transfert obtenues à

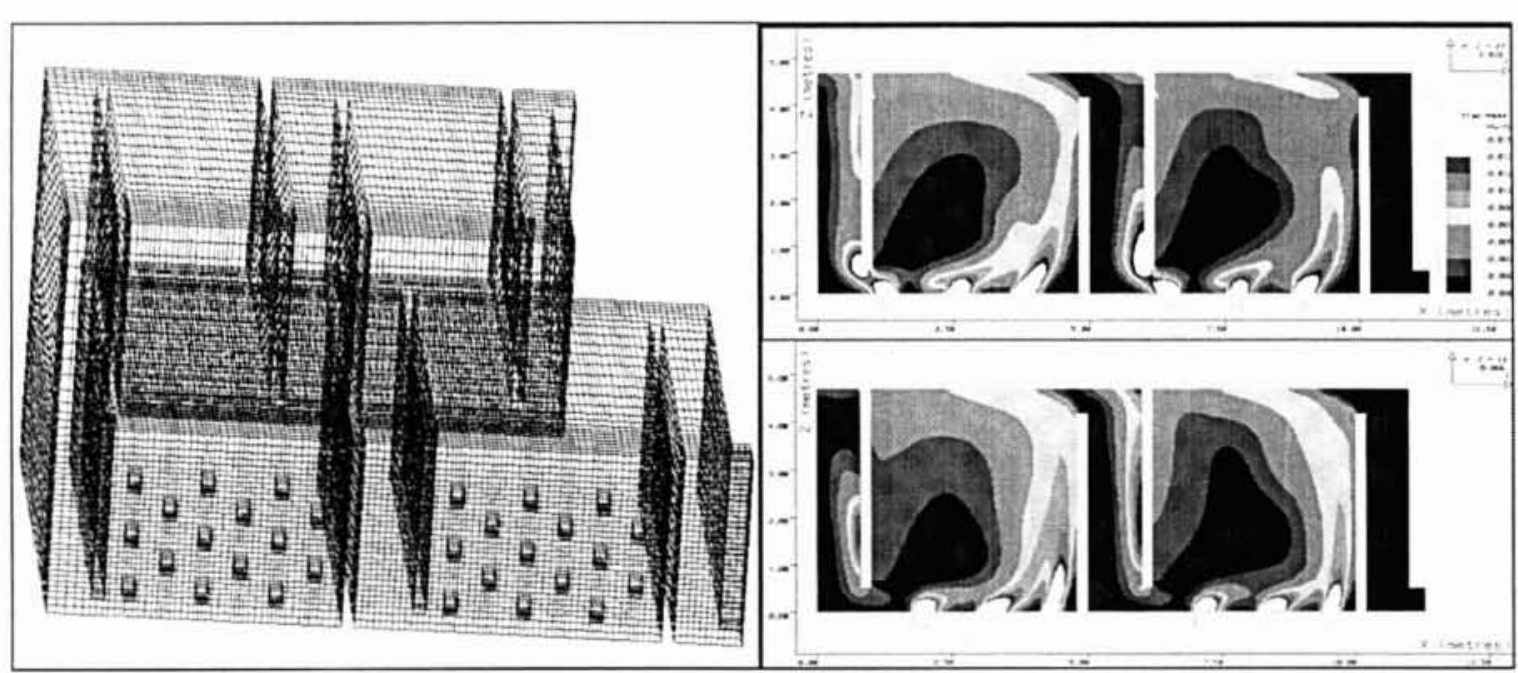

8. Maillage numérique 3D du réacteur d'ozonation et distribution de la rétention gazeuse dans deux plans verticaux du système.

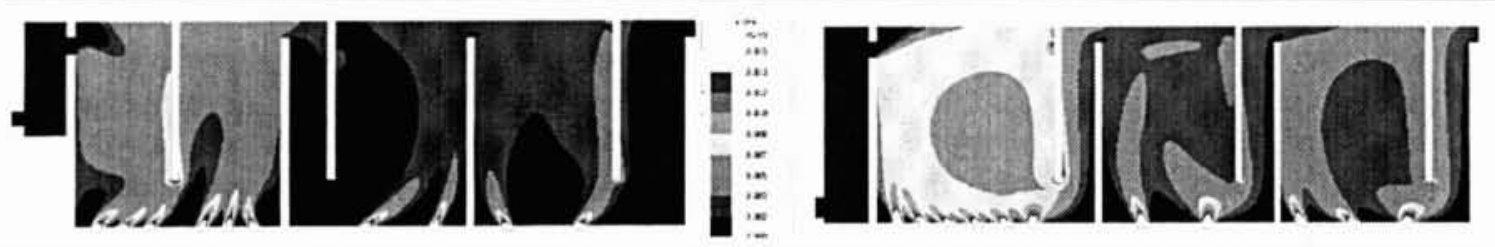

9. Distribution de la fraction volumique du gaz dans le réacteur (coupes verticales). 
l'échelle pilote ou industrielle dans différentes conditions de fonctionnement permettent d'optimiser à la fois la position de diffuseurs et leur débit respectif. Des traçages numériques ont été réalisés afin d'obtenir les courbes de distribution des temps de séjour et quantifier le coefficient de dispersion dans le système. Le traçage au chlorure de lithium $(\mathrm{LiCl})$ effectué sur le réacteur industriel a été utilisé pour valider les prédictions du modèle numérique.

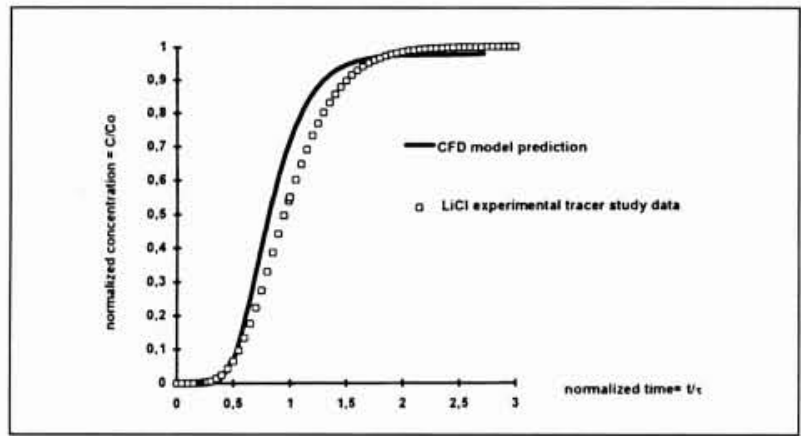

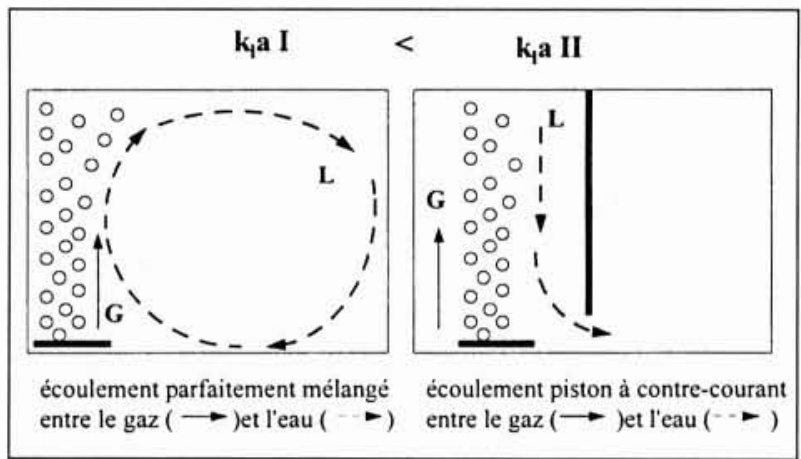

10. Courbe de Distribution des Temps de séjour dans le réacteur d'ozonation.

\section{IV $\square$ CONCLUSION}

La prédiction du transfert de masse dans les réacteurs diphasiques nécessite une bonne connaissance de l'hydrodynamique du réacteur et l'outil de la Mécanique des Fluides Numérique est un plus pour comprendre son fonctionnement. Le code ASTRID, utilisé en parallèle à des mesures expérimentales permet de simuler de façon satisfaisante les divers réacteurs diphasiques gaz-liquide et permet d'obtenir de très bons résultats quantitatifs dans le cas de l'airlift et aide à comprendre de manière plus qualitative l'hydrodynamique des bassins d'aération et des réacteurs d'ozonation. Cependant, dans l'utilisation des outils de la Mécanique des Fluides Numérique il est primordial de connaître la physique du problème pour, d'une part poser des hypothèses simplificatrices correctes et d'autre part, donner des relations de fermeture adéquates. Les différents types de contacteurs gaz-liquide, jusqu'alors conçus à partir de corrélations empiriques, peuvent être maintenant étudiés, améliorés ou dimensionnés à l'aide de la simulation numérique.

\section{RÉFÉRENCES}

[1] Chisti M.Y. (1989). - Air-lift Bioreactors, ed. by Elsevier Sci.Publ., London.

[2] Clift R., Grace J.R. and Weber M.E. (1978). - Bubbles, Drops and Particles, Academic Press.
[3] Cockx A., Liné A., Roustan M. and Do Quang Z. (1997,a). - Numerical simulation and physical modeling of the hydrodynamics in an air-lift internal loop reactor, $G L S^{\prime} 97$, December 7-10, Kanagawa, Japan.

[4] Cockx A., Liné A., Roustan M. \& Hébrard G. (1997,b). Simulation numérique et validation expérimentale de la dispersion axiale dans des contacteurs gaz-liquide, $\sigma^{e}$ Congrès Français de Génie des Procédés, Paris, 24-26 septembre, France.

[5] Cockx A., Liné A., Roustan M. \& Do Quang Z. (1997,c). Simulation numérique de différents réacteurs gaz-liquide, $\sigma^{e}$ Congrès Français de Génie des Procédés, Paris, 24-26 septembre, France.

[6] Cockx A., Bugay S., Liné A. and Roustan M. (1998). - PIV measurements and numerical simulations of the hydrodynamics induced by a screen of air bubbles, submitted to the Third International Conference on Multiphase Flow, ICMF'98, Lyon, June 8-12, France.

[7] Couvert A., Hébrard G., Liné A., Roustan M., Lazarova V. and Do QuANG Z. (1997). - Etude hydrodynamique et modélisation d'un réacteur de type air-lift à paroi interne, $\sigma^{e}$ Congrès Français de Génie des Procédés, Paris, 24-26 septembre.

[8] Deutch E. \& Simonin O. (1991). - Large eddy simulation applied to the motion of particles in stationary homogeneous fluid trubulence, Turbulence Modification in Multiphase Flows, Proceedings of the first ASME/JSME Fluid Engineering Conf, Michaelides E.E., Fukano T. \& Serizawa A., FED 110, pp. 35 42.

[9] IsHII M. and ZUBER N. (1979). - Drag coefficient and relative velocity in bubbly, droplet or particulate flows, AIChE J., 25(5), 843-855.

[10] Karamanev D.G. (1994). - Rise of gas bubbles in quiescent liquids, AIChE J., 40(8), 1418-1421.

[11] Lazarova V., Do Quang Z., Cockx A., Hébrard G., CouVert A., Liné A., Roustan M. and BAstoul D. (1997). CFD modeling and experimental study of a new circulation bed bioreactor performances. International Conference on Bioreactor and Bioprocess Fluid Dynamics. BHR Group. Edinburgh 1-3 July, UK. Ed. A.W. Nienow. 251-260.

[12] MOORE D.W. (1963). - The boundary layer on a spherical gas bubble, J. Fluid Mech., 16, 161-176.

[13] Moore D.W. (1965), - The velocity of rise of distroted gas bubbles in a liquid of small viscosity, J. Fluid Mech., 23 (4), 749-766.

[14] Roustan M. and LinÉ A. (1996). - Quels sont les critères d'extrapolation pour les systèmes d'aération. Tribune de l'Eau, 49 (583-584), 53-58.

[15] Simonin O. \& Viollet P.L. (1988), - On the computation of turbulent two-phase flows in the eulerian formulation, Turbulent Two-Phase System, Euromech 234, Toulouse, France.

[16] Simonin O. \& Viollet P.L. (1989), - Numerical study of phase dispersion mechanisms in turbulent bubbly flows, International Conference on Mechanics of Two-Phase Flows, 12-15 June, Taipei, Taiwan.

[17] Do-Quang Z. (1993). - Etudes expérimentale et numérique des performances des contacteurs de désinfection de l'eau par le chlore, Thèse de l'Institut National des Sciences Appliquées de Toulouse, France.

[18] Thai Van D., Minier J.P., Simonin O., Freydier P., Olive J. (1994). - Multidimensional two-phase model computation of turbulent dispersed two-phase flows, Proceedings of the ASME Fluids Engineering Division Summer Meeting, Crowe C.T., Johnson R., Prosperetti A., Sommerfeld M. \& Tsuji Y., Eds, 1994 June 19-23, Lake Tahoe, USA, FED-Vol. 185, pp. 277-291. 